\title{
The numerical simulation of convection delayed dominated diffusion equation
}

\author{
P. Murali Mohan Kumar ${ }^{1, a}$, A.S.V. Ravi Kanth ${ }^{1}$ \\ ${ }^{1}$ Department of Mathematics, National Institute of Technology Kurukshetra, Haryana - 136119, India
}

\begin{abstract}
In this paper, we propose a fitted numerical method for solving convection delayed dominated diffusion equation. A fitting factor is introduced and the model equation is discretized by cubic spline method. The error analysis is analyzed for the consider problem. The numerical examples are solved using the present method and compared the result with the exact solution.
\end{abstract}

\section{Introduction}

Consider the following convection delayed dominated diffusion equation

$\varepsilon y^{\prime \prime}(x)+a(x) y^{\prime}(x)+b(x) y(x-\delta)=0$ on $\Omega=[0,1]$

subject to the interval conditions

$y(x)=\phi(x)$ on $\delta \leq x \leq 0, y(1)=\gamma$

where $0<\varepsilon<<1$ is a perturbation parameter and $\delta$ is a small shifting parameter of order $\varepsilon$. It is also assumed that $a(x), b(x), \varphi(x)$ are smooth functions and $\gamma$ is a constant. These convection diffusion delayed types with dominated convection term problems play an important role in the mathematical modelling of various practical phenomena in the engineering and environmental sciences, for examples include high Reynold's number flow in fluid dynamics, heat transport problems with large Pecklet number, modelling the problems in mathematical biology and semi-conductor devices etc. It is challenging to develop efficient numerical methods for solving convection diffusion with dominated convection term due to the existence of boundary layers. Standard discretization methods for solving such kind of problems are unstable and fails to give accurate results when perturbation parameter $\varepsilon$ is small. Therefore, it is challenging to develop suitable numerical methods to these problems, whose accuracy does not depend on the parameter $\varepsilon$. Lange and Miura[1] initiated the singular perturbation analysis of boundary value problems for differential difference equations with small shifts. The numerical study of second order singularly perturbed delay differential equations has been given in [2-3] and references therein.

In this paper, we present an exponentially fitted method on uniform mesh based on cubic spline method for the convection delayed dominated diffusion equation. The layout of the paper is organized as follows: Continuation of the problem is presented in next section and follows

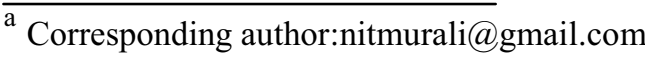

description of the method. In Section 3, the error analysis of the method is discussed. Section 4 ends with the Numerical results.

\section{Continuous problem}

An application of Taylor series in (1) yields $\varepsilon y^{\prime \prime}(x)+(a(x)-\delta b(x)) y^{\prime}(x)+b(x) y(x)=0, \quad x_{i-1}<x<x_{i+1}$ $y(x)=\phi_{0}, y(1)=\gamma$

We assume that $(a(x)-\delta b(x)) \geq M>0$ throughout the interval $[0,1]$, where $M$ is positive constant, then the problem (3) exhibits boundary layer at $x=0$. From the theory of singular perturbations [4],

$$
y(x)=y_{0}(x)+\left(\alpha-y_{0}(0)\right) e^{\frac{-(a(0)-\delta b(0))}{\varepsilon} x}+o(\varepsilon)
$$

where $y_{0}(x)$ is the solution of the reduced problem given by

$$
(a(x)-\delta b(x)) y_{0}^{\prime}(x)+b(x) y_{0}(x)=0 \text { with } y_{0}(1)=\beta
$$

From Eq.(4) as $h \rightarrow 0$, we obtain

$\lim _{h \rightarrow 0} y(i h)=y_{0}(0)+\left(\phi_{0}-y_{0}(0)\right) e^{\frac{-(a(0)-\delta b(0))}{\varepsilon} i h}$

Let $\rho=\frac{h}{\varepsilon}$, then

$\lim _{h \rightarrow 0} y(i h)=y_{0}(0)+\left(\phi_{0}-y_{0}(0)\right) e^{-(a(0)-\delta b(0)) i \rho}$

Now introducing an exponentially fitting factor $\sigma(\rho)$ to the Eq.(3), we get

$$
\sigma(\rho) y^{\prime \prime}(x)+(a(x)-\delta b(x)) y^{\prime}(x)-b(x) y(x)=0
$$

with $\quad \mathrm{y}(0)=\phi_{0}, y(1)=\gamma$ 
The fitting factor $\sigma(\rho)$ is to be determined in such a way that the solution of Eq.(6) converges uniformly to the solution Eq.(3) .

Lemma 1. Let $u(x)$ be a smooth function satisfing the $u(0) \geq 0, u(1) \geq 0 \quad$ and $\quad L u(x) \leq 0, \forall x \in \Omega$, then $u(x) \geq 0, \forall x \in \check{\Omega}$.

Prof We can prove the above lemma by method of contradiction. Let $x^{*} \in \check{\Omega}$ be such that $u\left(x^{*}\right)=\min u(x), x \in \check{\Omega}$ and assume that $u\left(x^{*}\right)<0$. Clearly $x^{*} \notin\{0,1\}$ and $u^{\prime}\left(x^{*}\right)=0$ and $u^{\prime \prime}\left(x^{*}\right) \geq 0$. Now consider

$L u\left(x^{*}\right) \equiv \epsilon u^{\prime \prime}\left(x^{*}\right)+\left(a\left(x^{*}\right)-\delta b\left(x^{*}\right)\right) u^{\prime}\left(x^{*}\right)+b\left(x^{*}\right) u\left(x^{*}\right)>0$

Which is contradiction to our assumption. Hence $u(x) \geq 0, \forall x \in \check{\Omega}$.

Lemma 2. Let $u(x)$ is the solution of the boundary value problem (3), then

$\|u\| \leq \max \left\{\left|\phi_{0}\right|,|\gamma|\right\}$

Proof. Let $\psi^{ \pm}(x)$ be two barrier functions defined by

$\psi \pm(x)=\max \left\{\left|\phi_{0}\right|,|\gamma|\right\} \pm u(x)$

Then, we have

$\psi \pm(0)=\max \left\{\left|\phi_{0}\right|,|\gamma|\right\} \pm u(0) \geq 0$

$\psi \pm(1)=\max \left\{\left|\phi_{0}\right|,|\gamma|\right\} \pm u(1) \geq 0$

and

$L_{\tau} \psi^{ \pm}(x)=\epsilon \psi^{ \pm^{\prime \prime}}(x)+(a(x)-\delta b(x)) \psi^{ \pm^{\prime}}(x)+b(x) \psi^{ \pm}(x)$

$= \pm L_{\tau} u(x)+b(x) \max \left\{\left|\phi_{0}\right|,|\gamma|\right\}$

$\leq b(x) \max \left\{\left|\phi_{0}\right|,|\gamma|\right\}$ as $b(x) \leq-\theta<0$.

Using this inquality in the above inequality, we get

$L_{\tau} \psi^{ \pm}(x) \leq b(x) \max \left\{\left|\phi_{0}\right|,|\gamma|\right\} \leq 0 \forall x \in(0,1)$

Therefore by the maximum principle[5], we obtain $\psi^{ \pm}(x) \geq 0, \forall x \in[0,1]$, which gives the required estimate.

\subsection{Description of the method}

Let $x_{0}=0, x_{n}=1, x_{i}=i h, h=\frac{1}{N}$. The spline function $S(x, \tau)=S(x)$ satisfying in the interval $\left[x_{i}, x_{i+1}\right]$ and the differential equation

$$
\begin{aligned}
S^{\prime \prime}(x)-\tau S(x) & =\left[S^{\prime \prime}\left(x_{i}\right)-\tau S\left(x_{i}\right)\right]\left(\frac{x_{i+1}-x}{h}\right) \\
& +\left[S^{\prime \prime}\left(x_{i+1}\right)-\tau S\left(x_{i+1}\right)\right]\left(\frac{x-x_{i}}{h}\right)
\end{aligned}
$$

Where $S\left(x_{i}\right)=y_{i}$ and $\tau>0$ is termed as cubic spline in compression. Following Aziz and Khan [6], we obtain the tridiagonal system

$$
h^{2}\left(\lambda_{1} M_{i-1}+2 \lambda_{2} M_{i}+\lambda_{1} M_{i+1}\right)=y_{i-1}-2 y_{i}+y_{i+1}
$$

Where

$$
\begin{aligned}
& \lambda_{1}=\frac{1}{\lambda^{2}}\left(1-\frac{\lambda}{\sin \lambda}\right), \lambda_{2}=\frac{1}{\lambda^{2}}(\lambda \cot \lambda-1), \\
& M_{i}=y^{\prime \prime}\left(x_{i}\right), i=1(1) N-1 .
\end{aligned}
$$

Substituting

$$
\begin{gathered}
\sigma(\rho) \varepsilon M_{j}=-\left(a\left(x_{j}\right)-\delta b\left(x_{j}\right)\right) y^{\prime}\left(x_{j}\right)-b\left(x_{j}\right) y\left(x_{j}\right),{ }_{\mathrm{i}} \\
j=i, i \pm 1,
\end{gathered}
$$

$\mathrm{n}$ Eq.(10) and using the following three point approximations for first order derivatives

$$
\begin{gathered}
y_{i}^{\prime}=\frac{y_{i+1}-y_{i-1}}{2 h} \\
y_{i+1}^{\prime}=\frac{3 y_{i+1}-4 y_{i}+y_{i-1}}{2 h} \\
y_{i-1}^{\prime}=\frac{-y_{i+1}+4 y_{i}-3 y_{i-1}}{2 h}
\end{gathered}
$$

We get the following difference scheme

$$
E_{i} y_{i-1}-F_{i} y_{i}+G_{i} y_{i+1}=0, i=1(1) N-1
$$

Where

$E_{i}=\frac{\sigma}{\rho}-\frac{3 \lambda_{1}}{2}\left(a_{i-1}-\delta b_{i-1}\right)-\lambda_{2}\left(a_{i}-\delta b_{i}\right)+\frac{\lambda_{2}}{2}\left(a_{i+1}-\delta b_{i+1}\right)+\lambda_{1} h b_{i-1}$

$$
F_{i}=\frac{2 \sigma}{\rho}-2 \lambda_{1}\left(a_{i-1}-\delta b_{i-1}\right)+2 \lambda_{2}\left(a_{i+1}-\delta b_{i+1}\right)+2 \lambda_{2} h b_{i}
$$

$G_{i}=\frac{\sigma}{\rho}-\frac{\lambda_{1}}{2}\left(a_{i-1}-\delta b_{i-1}\right)+\lambda_{2}\left(a_{i}-\delta b_{i}\right)+\frac{3 \lambda_{2}}{2}\left(a_{i+1}-\delta b_{i+1}\right)+\lambda_{1} h b_{i+1}$

Eq.(11) gives a system of $N-1$ equations with $N+1$ unknowns. These $N-1$ equations together with the

Eq.(7) are sufficient to solve the system by using Thomas algorithm.

\subsection{Determination of fitting factor}

Taking the limit as $h \rightarrow 0$ in Eq.(11), we obtain

$$
\begin{aligned}
& \left(\frac{\sigma}{\rho}-(a(0)-\delta b(0))\left(\lambda_{1}+\lambda_{2}\right)\right) \lim _{h \rightarrow 0} y_{i-1}-\frac{2 \sigma}{\rho} \lim _{h \rightarrow 0} y_{i} \\
& \left(\frac{\sigma}{\rho}+(a(0)-\delta b(0))\left(\lambda_{1}+\lambda_{2}\right)\right) \lim _{h \rightarrow 0} y_{i+1}=0
\end{aligned}
$$

Substituting Eq.(5) in Eq.(12) and then simplifying, we get the variable fitting factor as follows

$$
\sigma_{i}(\rho)=\frac{\rho}{2}\left(\lambda_{1}+\lambda_{2}\right)\left(a\left(x_{i}\right)-\delta b\left(x_{i}\right)\right) \operatorname{coth}\left(\frac{\left(a\left(x_{i}\right)-\delta b\left(x_{i}\right)\right) \rho}{2}\right)
$$

$$
\sigma(\rho)=\frac{\rho}{2}\left(\lambda_{1}+\lambda_{2}\right)(a(0)-\delta b(0)) \operatorname{coth}\left(\frac{(a(0)-\delta b(0)) \rho}{2}\right)
$$

is a constant fitting factor for left end boundary layer.

\section{Error analysis}

Substituting $\sigma \varepsilon M_{j}=-\left(a_{j}-\delta b_{j}\right) y_{j}^{\prime}-b_{j} y_{j}, j=i, i \pm 1$ in (10), we obtain 


$$
\begin{aligned}
\sigma \varepsilon\left(y_{i-1}\right. & \left.-2 y_{i}+y_{i+1}\right) \\
& =h^{2} \lambda_{1}\left(-\left(a_{i-1}-\delta b_{i-1}\right) y_{i-1}^{\prime}-b_{i-1} y_{i-1}\right) \\
& +2 \lambda_{2} h^{2}\left(-\left(a_{i}-\delta b_{i}\right) y_{i}^{\prime}-b_{i} y_{i}\right) \\
& +h^{2} \lambda_{1}\left(-\left(a_{i+1}-\delta b_{i+1}\right) y_{i+1}^{\prime}-b_{i+1} y_{i+1}\right)
\end{aligned}
$$

Putting exact solution in (14), we get

$$
\begin{aligned}
\sigma \varepsilon\left(y\left(x_{i-1}\right)-2 y\left(x_{i}\right)+y\left(x_{i+1}\right)\right) \\
\quad=h^{2} \lambda_{1}\left(-\left(a_{i-1}-\delta b_{i-1}\right) y^{\prime}\left(x_{i-1}\right)-b_{i-1} y\left(x_{i-1}\right)\right) \\
+2 \lambda_{2} h^{2}\left(-\left(a_{i}-\delta b_{i}\right) y^{\prime}\left(x_{i}\right)-b_{i} y\left(x_{i}\right)\right) \\
+h^{2} \lambda_{1}\left(-\left(a_{i+1}-\delta b_{i+1}\right) y^{\prime}\left(x_{i+1}\right)-b_{i+1} y\left(x_{i+1}\right)\right)
\end{aligned}
$$

Where

$$
\begin{gathered}
T_{i o}(h)=\frac{h^{4}}{12} \sigma \varepsilon\left(1-12 \lambda_{1}\right) y^{(4)}\left(\xi^{i}\right)+\frac{h^{6}}{360} \sigma \varepsilon\left(1-30 \lambda_{1}\right) y^{(6)}\left(\xi^{i}\right) \\
x_{i-1}<\xi^{i}<x_{i+1}
\end{gathered}
$$

for any choice of $\lambda_{1}$ and $\lambda_{2}$ whose sum is $1 / 2$. Subtracting Eq.(14) from Eq.(15) and substituting $e_{j}=y\left(x_{j}\right)-y_{j}, j=i, i \pm 1$, we get

$\left(\sigma \varepsilon+\lambda_{1} h^{2} b_{i-1}\right) e_{i-1}+\left(-2 \sigma \varepsilon+2 \lambda_{2} h^{2} b_{i}\right) e_{i}+\left(\sigma \varepsilon+\lambda_{1} h^{2} b_{i+1}\right) e_{i+1}=$ $-h^{2}\left[\lambda_{1}\left(a_{i-1}-\delta b_{i-1}\right) e_{i-1}^{\prime}-2 \lambda_{2}\left(a_{i}-\delta b_{i}\right) e_{i}^{\prime}-\lambda_{1}\left(a_{i+1}-\delta b_{i+1}\right) e_{i+1}^{\prime}\right]$ $+T_{i o}(h)$

we have

$$
\begin{aligned}
e_{i-1}^{\prime} & =y^{\prime}\left(x_{i-1}\right)-y_{i-1}^{\prime} \\
& =\frac{h^{2}}{3} y^{(3)}\left(x_{i}\right)-\frac{h^{2}}{12} y^{(4)}\left(x_{i}\right)+\frac{h^{4}}{30} y^{(5)}\left(\xi_{1}^{i}\right) \\
e_{i+1}^{\prime} & =y^{\prime}\left(x_{i+1}\right)-y_{i+1}^{\prime} \\
& =\frac{h^{2}}{3} y^{(3)}\left(x_{i}\right)+\frac{h^{2}}{12} y^{(4)}\left(x_{i}\right)+\frac{h^{4}}{30} y^{(5)}\left(\xi_{2}^{i}\right) \\
e_{i}^{\prime} & =y^{\prime}\left(x_{i}\right)-y_{i}^{\prime} \\
& =-\frac{h^{2}}{6} y^{(3)}\left(x_{i}\right)-\frac{h^{4}}{120} y^{(5)}\left(\xi_{3}^{i}\right)
\end{aligned}
$$

$$
x_{i-1}<\xi_{1}^{i}, \xi_{2}^{i}, \xi_{3}^{i}<x_{i+1}
$$

Using Eqs.(18)-(20) in Eq.(17), we get

$$
\begin{aligned}
& \left(\sigma \varepsilon+\lambda_{1} h^{2} b_{i-1}\right) e_{i-1}+\left(-2 \sigma \varepsilon+2 \lambda_{2} h^{2} b_{i}\right) e_{i}+\left(\sigma \varepsilon+\lambda_{1} h^{2} b_{i+1}\right) e_{i+1}= \\
& -\frac{h^{4}}{3}\left[\lambda_{1}\left(a_{i-1}-\delta b_{i-1}\right)-2 \lambda_{2}\left(a_{i}-\delta b_{i}\right)+\lambda_{1}\left(a_{i+1}-\delta b_{i+1}\right)\right] y^{(3)}\left(x_{i}\right) \\
& +\frac{h^{5}}{12} \lambda_{1}\left[\left(a_{i-1}-\delta b_{i-1}\right)-\left(a_{i+1}-\delta b_{i+1}\right)\right] y^{(4)}\left(x_{i}\right) \\
& -\frac{h^{6}}{30} \lambda_{1}\left[\left(a_{i-1}-\delta b_{i-1}\right) y^{(5)}\left(\xi_{1}\right)+\left(a_{i+1}-\delta b_{i+1}\right) y^{(5)}\left(\xi_{2}\right)\right] \\
& +\frac{h^{6}}{60} \lambda_{2}\left(a_{i}-\delta b_{i}\right) y^{(5)}\left(\xi_{3}\right)+T_{i 0}(h)
\end{aligned}
$$

$$
\begin{aligned}
& a_{i-1}=a_{i}-h a_{i}^{\prime}+\frac{h^{2}}{2 !} a_{i}^{(2)}\left(\eta_{1}\right) \\
& a_{i+1}=a_{i}+h a_{i}^{\prime}+\frac{h^{2}}{2 !} a_{i}^{(2)}\left(\eta_{2}\right) \\
& b_{i-1}=b_{i}-h b_{i}^{\prime}+\frac{h^{2}}{2 !} b_{i}^{(2)}\left(\eta_{3}\right) \\
& b_{i+1}=b_{i}+h b_{i}^{\prime}+\frac{h^{2}}{2 !} b_{i}^{(2)}\left(\eta_{4}\right)
\end{aligned}
$$

Where $x_{i-1}<\eta_{1}, \eta_{2}, \eta_{3}, \eta_{4}<x_{i+1}$

Using these expansions and Eq.(21), we have $\left(\sigma \varepsilon+\lambda_{1} h^{2} b_{i-1}\right) e_{i-1}+\left(-2 \sigma \varepsilon+2 \lambda_{2} h^{2} b_{i}\right) e_{i}$

$+\left(\sigma \varepsilon+\lambda_{1} h^{2} b_{i+1}\right) e_{i+1}=T_{i}(h)$

Where

$$
\begin{aligned}
T_{i}(h)= & -\frac{h^{4}}{3}\left(a_{i}-\delta b_{i}\right) y^{(3)}\left(x_{i}\right)+\frac{h^{4}}{12}\left(1-12 \lambda_{1}\right) \varepsilon \sigma y^{(4)}\left(x_{i}\right) \\
& -\frac{h^{6}}{6} \lambda_{1}\left(a_{i}^{\prime}-\delta b_{i}^{\prime}\right) y^{(4)}\left(x_{i}\right)+O\left(h^{6}\right)
\end{aligned}
$$

Clearly, it can be seen that $T_{i}(h)=O\left(h^{4}\right)$ for the choice of $\lambda_{1}$ and $\lambda_{2}$ whose sum is $1 / 2$.

Let $J=\operatorname{trid}\left[\begin{array}{cll}\sigma \varepsilon & -2 \sigma \varepsilon & \sigma \varepsilon\end{array}\right], \quad D=\operatorname{trid}\left[\begin{array}{lll}\lambda_{1} & 2 \lambda_{2} & \lambda_{1}\end{array}\right]$ are $(N-1) \times(N-1) \quad$ tridiagonal matrices and $Q=\left(b_{1}, b_{2}, \cdots, b_{N-1}\right)^{t} \quad$ and $\quad E=\left(e_{1}, e_{2}, \cdots, e_{N-1}\right)^{t} \quad$ are $(N-1)$ component vectors. Eq. (21) can be written in matrix vector form as

$A E=T_{i}(h), \quad$ where $A=\left(J-h^{2} D Q\right)$

Clearly, the row sums $S_{1}, S_{2}, \cdots, S_{N-1}$ of $A$ are

$S_{1}=-\sigma_{1} \varepsilon+2 \lambda_{2} h^{2} b_{1}+\lambda_{1} h^{2} b_{2}$

$S_{i}=h^{2}\left(\lambda_{1} b_{i-1}+2 \lambda_{2} b_{i}+\lambda_{1} b_{i+1}\right)=h^{2} B_{i}, i=2(1) N-2$

$S_{N-1}=-\sigma_{N-1} \varepsilon+\lambda_{1} h^{2} b_{N-2}+2 \lambda_{2} h^{2} b_{N-1}$

For sufficiently small $h$, the matrix $A$ is irreducible and monotone which implies $A^{-1}$ exists. Hence from Eq.(24), we have $E=A^{-1} T_{i}(h)$.

From the theory of matrices we have,

$$
\sum_{i=1}^{N-1} \bar{p}_{k, i} S_{i}=1, \quad k=1(1) N-1
$$

Where $\bar{p}_{k, i}$ is the $(k, i)^{t h}$ element of the matrix $A^{-1}$. Therefore

$$
\begin{gathered}
\sum_{i=1}^{N-1} \bar{p}_{k, i} \leq \frac{1}{\min _{1 \leq i \leq N-1} S_{i}}=\frac{1}{h^{2} B_{i}} \leq \frac{1}{h^{2}\left|B_{i}\right|} \\
E=\sum_{i=1}^{N-1} \bar{p}_{k, i} T_{i}(h), k=1(1) N-1
\end{gathered}
$$

Therefore

$$
\|E\|=\frac{K h^{2}}{\left|B_{i}\right|}
$$

Where $K$ is constant independent of $h$. Therefore $\|E\|=O\left(h^{2}\right)$ for the choice of parameters $\lambda_{1}$ and $\lambda_{2}$ 
whose sum is $1 / 2$. From the Eq.(25), it is observed that the proposed method is $\varepsilon$ uniform convergent since the error is of order of the form $\|E\|=K^{*} h^{2}$, where $K^{*}$ is independent of perturbation parameter $\varepsilon$.

\section{Numerical Results}

To demonstrate the applicability of the method, we consider two convective diffusion with dominated convection term exhibiting boundary layer at left end of the interval and one right end of the interval. The exact solution of the boundary value problem (1) with constant coefficients is given by

$$
y(x)=\frac{\gamma-\phi e^{m_{2}}}{e^{m_{1}}-e^{m_{2}}} e^{m_{1} x}+\frac{\phi e^{m_{1}}-\gamma}{e^{m_{1}}-e^{m_{2}}} e^{m_{2} x}
$$

where

$$
\begin{aligned}
& m_{1}=\frac{-(a-\delta b)+\sqrt{(a-\delta b)^{2}-4 \varepsilon b}}{2 \varepsilon} \\
& m_{2}=\frac{-(a-\delta b)-\sqrt{(a-\delta b)^{2}-4 \varepsilon b}}{2 \varepsilon}
\end{aligned}
$$

\section{Example 1.}

Consider the following left end layer singularly perturbed of convection delayed dominated diffusion

$\varepsilon y^{\prime \prime}(x)+5 y^{\prime}(x)+y(x-\delta)=0$ on $\Omega=[0,1]$

subject to the interval conditions

$y(x)=1$ on $\delta \leq x \leq 0, y(1)=0$

In Table 1, represents the comparison between the present method and the method in [7] with fixed $\varepsilon$ and $\delta$. It is observed that the present method gives more accurate results than the method in [7]. Table 2 indicates the maximum absolute error for different $N$ and $\varepsilon$, it is observed from the results, the method is $\varepsilon$-uniform convergent. Figure 1 displays the numerical solution for different $\varepsilon$ values, the solution of the problem exhibits the boundary layer behavior to the left side while the perturbation parameter tends to zero.

Table 1. Numerical solution for Example 1. with $\varepsilon=2^{-2}$ and $\delta=0.1, \lambda_{1}=1 / 12, \lambda_{2}=5 / 12$

\begin{tabular}{cccc}
\hline $\mathbf{x}$ & Exact & Proposed & Method in [7] \\
\hline 0.1 & 0.14379381821116 & 0.14380334377735 & 0.14085841827929 \\
0.2 & 0.02067665891105 & 0.02067939843477 & 0.01984109173050 \\
0.3 & 0.00297317208765 & 0.00297376299483 & 0.00279478220875 \\
0.4 & 0.00042752012899 & 0.00042763342218 & 0.00039366596699 \\
0.5 & 0.00006147117862 & 0.00006149154060 & 0.00005544852472 \\
0.6 & 0.00000883567395 & 0.00000883918533 & 0.00000780774988 \\
0.7 & 0.00000126708504 & 0.00000126767192 & 0.00000109714556 \\
0.8 & 0.00000017883874 & 0.00000017893301 & 0.00000015190043 \\
0.9 & 0.00000002242424 & 0.00000002243736 & 0.00000001875470 \\
\hline
\end{tabular}

Table 2. Maximum absolute errors for the Example 1. $\lambda_{1}=1 / 12, \lambda_{2}=5 / 12$

\begin{tabular}{lcccc}
\hline$\varepsilon \downarrow N \rightarrow$ & $\mathbf{1 2 8}$ & $\mathbf{2 5 6}$ & $\mathbf{5 1 2}$ & $\mathbf{1 0 2 4}$ \\
\hline $2^{-1}$ & $3.90 \mathrm{E}-06$ & $9.76 \mathrm{E}-07$ & $2.44 \mathrm{E}-07$ & $6.10 \mathrm{E}-08$ \\
$2^{-2}$ & $7.65 \mathrm{E}-06$ & $1.91 \mathrm{E}-06$ & $4.78 \mathrm{E}-07$ & $1.19 \mathrm{E}-07$ \\
$2^{-3}$ & $1.52 \mathrm{E}-05$ & $3.78 \mathrm{E}-06$ & $9.46 \mathrm{E}-07$ & $2.36 \mathrm{E}-07$ \\
$2^{-4}$ & $3.04 \mathrm{E}-05$ & $7.55 \mathrm{E}-06$ & $1.88 \mathrm{E}-06$ & $4.70 \mathrm{E}-07$ \\
$2^{-8}$ & $1.33 \mathrm{E}-04$ & $6.55 \mathrm{E}-05$ & $2.77 \mathrm{E}-05$ & $8.29 \mathrm{E}-06$ \\
$2^{-12}$ & $1.33 \mathrm{E}-04$ & $6.65 \mathrm{E}-05$ & $3.32 \mathrm{E}-05$ & $1.66 \mathrm{E}-05$ \\
$2^{-16}$ & $1.33 \mathrm{E}-04$ & $6.65 \mathrm{E}-05$ & $3.32 \mathrm{E}-05$ & $1.66 \mathrm{E}-05$ \\
$2^{-32}$ & $1.33 \mathrm{E}-04$ & $6.65 \mathrm{E}-05$ & $3.32 \mathrm{E}-05$ & $1.66 \mathrm{E}-05$ \\
\hline
\end{tabular}

\section{Example 2.}

Consider the following variable coefficient of left end layer singularly perturbed problem

$$
\varepsilon y^{\prime \prime}(x)+\left(1-\frac{x}{2}\right) y^{\prime}(x)-\frac{1}{2} y(x)=0 \text { on } \Omega=[0,1]
$$

subject to the interval conditions

$$
y(x)=0 \text { on } \delta \leq x \leq 0, y(1)=1
$$

A valid solution for the above problem is given by

$$
u(x)=\frac{1}{2-x}-e^{\frac{-\left(x-\frac{x^{2}}{4}\right)}{\varepsilon}}
$$

Figure 2. indicates the numerical solution for different $\varepsilon$ and $\delta$ values, it is observed that the boundary layer behavior is not only on perturbation parameter and also depends on the delay parameter.

\subsection{Determination of fitting factor for right end problem}

We assume that $(a(x)-\delta b(x)) \leq M<0, M$ is a constant then, the Eq.(3) exhibits boundary layer at $x=1$.

$$
y(x)=y_{0}(x)+\left(\gamma-y_{0}(1)\right) e^{\frac{-(a(1)-\delta b(1))}{\varepsilon}(1-x)}+o(\varepsilon)
$$

As $h \rightarrow 0$, Eq.(26) becomes

$$
\lim _{h \rightarrow 0} y(i h)=y_{0}(x)+\left(\gamma-y_{0}(1)\right) e^{-(a(1)-\delta b(1))\left(\frac{1}{\varepsilon}-i \rho\right)}
$$

Introducing $\sigma(\rho)$ in Eq.(3) and by applying the same procedure as in Section(2) and simplifying, we get

$$
\begin{aligned}
& \sigma_{i}(\rho)=\frac{\rho}{2}\left(\lambda_{1}+\lambda_{2}\right)\left(a\left(x_{i}\right)-\delta b\left(x_{i}\right)\right) \operatorname{coth}\left(\frac{\left(a\left(x_{i}\right)-\delta b\left(x_{i}\right)\right) \rho}{2}\right) \\
& \sigma(\rho)=\frac{\rho}{2}\left(\lambda_{1}+\lambda_{2}\right)(a(1)-\delta b(1)) \operatorname{coth}\left(\frac{(a(1)-\delta b(1)) \rho}{2}\right)
\end{aligned}
$$

is a constant fitting factor for right end boundary value problem. 


\section{Example 3.}

Finally we consider the right end layer singularly perturbed of convection diffusion problem $\varepsilon y^{\prime \prime}(x)-5 y^{\prime}(x)+2 y(x-\delta)=0$ on $\Omega=[0,1]$ subject to the interval conditions $y(x)=0$ on $\delta \leq x \leq 0, y(1)=2$

Table 3 gives the comparison between present method and the method in [7] for fixed $\varepsilon$ and $\delta$. It is observed that the method yields results better than the existing method in [7]. Figure 2 indicates the numerical solution for different $\varepsilon$ and $\delta$ values. The perturbation parameter tends to zero the solution of the problem exhibits boundary layer behavior to the right side of the interval.

\section{Acknowledgements}

Authors would like to thank National Board for Higher Mathematics (NBHM), Government of India for providing financial support under the grant number 2/48(12)/2013/NBHM(R.P.)/R\&D II/1084.

Table 3. Numerical solution for Example 2. with $\varepsilon=2^{-2}$ and $\delta=0.1 \lambda_{1}=1 / 12, \lambda_{2}=5 / 12$

\begin{tabular}{cccc}
\hline $\mathbf{x}$ & Exact & Proposed & Method in [7] \\
\hline 0.1 & 0.00000001824795 & 0.00000001827085 & 0.00000001297418 \\
0.2 & 0.00000015942757 & 0.00000015960776 & 0.00000011682562 \\
0.3 & 0.00000124681219 & 0.00000124804968 & 0.00000094810121 \\
0.4 & 0.00000961694147 & 0.00000962512936 & 0.00000760202085 \\
0.5 & 0.00007404065246 & 0.00007409319019 & 0.00006086311380 \\
0.6 & 0.00056989545628 & 0.00057021895408 & 0.00048718987663 \\
0.7 & 0.00438637260011 & 0.00438823990526 & 0.00389970919703 \\
0.8 & 0.03376088858255 & 0.03377046939265 & 0.03121511401660 \\
0.9 & 0.25984953872717 & 0.25988640673333 & 0.24986042277630 \\
\hline
\end{tabular}

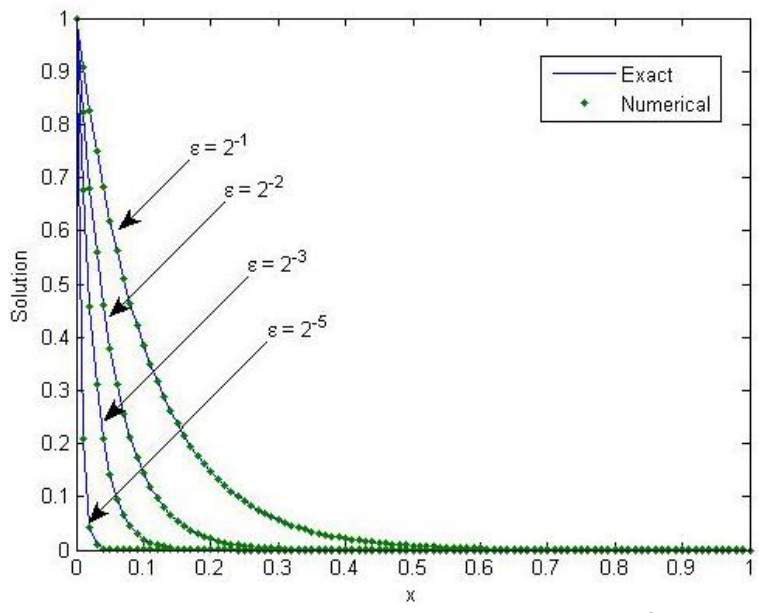

Figure 1. Numerical solution of the Example 1 for $\delta=0.1$

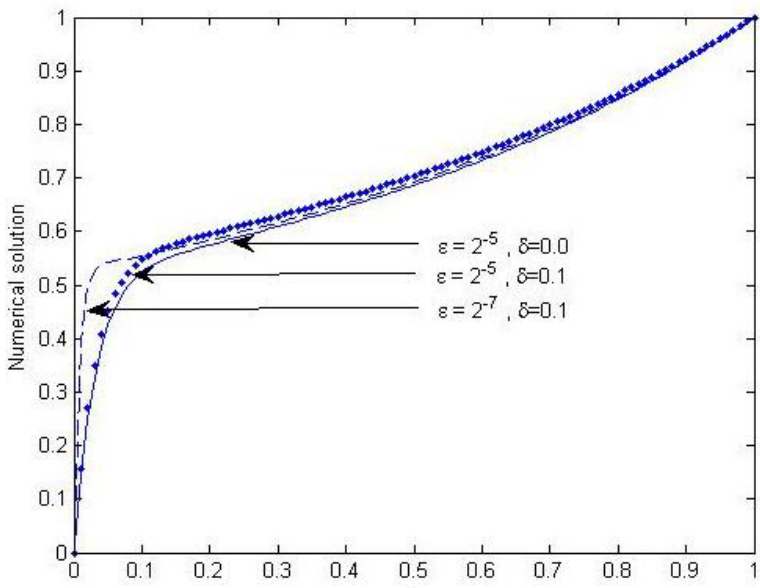

Figure 2. Numerical solution of the Example 2

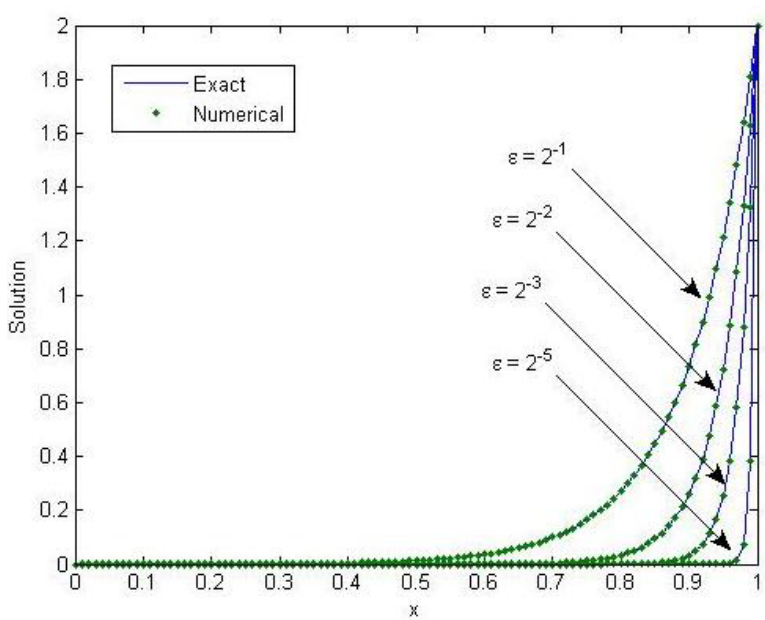

Figure3. Numerical solution of the Example 3 for $\delta=0.1$

\section{References}

1. C G. Lange, R M. Miura. SIAM Journal on Applied Mathematics 54(1) 249-272 (1994)

2. M. K. Kadalbajoo, K. K. Sharma. Applied Mathematics and Computation 157(1), 11-28 (2004)

3. M. K .Kadalbajoo, K. K. Sharma. Applied Mathematics and Computation 197(2), 692-707 (2008)

4. O’Malley Robert. Academic Press. (1974)

5. P.A. Farrell, A.F. Hegarty, J.J.H. Miller, E.O'Riordan, G.I. Shishkin, CRC Press LLC (2000)

6. T. Aziz, A. Khan, Journal of Computational and Applied Mathematics 147, 445-452 (2002)

7. M. Sharma, A. Kaushik, C. Li. Journal of Mathematical Chemistry 52(9), 2459-2474 (2014) 